The effects of sheared toroidal rotation on stability limits in tokamak plasmas

This article has been downloaded from IOPscience. Please scroll down to see the full text article.

2011 Plasma Phys. Control. Fusion 53125002

(http://iopscience.iop.org/0741-3335/53/12/125002)

View the table of contents for this issue, or go to the journal homepage for more

Download details:

IP Address: 128.178.125.184

The article was downloaded on 26/09/2012 at 11:20

Please note that terms and conditions apply. 


\title{
The effects of sheared toroidal rotation on stability limits in tokamak plasmas
}

\author{
I T Chapman ${ }^{1}, \mathbf{N}$ R Walkden ${ }^{1,2}$, J P Graves $^{3}$ and C Wahlberg $^{4}$ \\ ${ }^{1}$ Euratom/CCFE Fusion Association, Culham Science Centre, Abingdon, OX14 3DB, UK \\ 2 Physics Department, University of Bath, Bath, BA2 7AY, UK \\ ${ }^{3}$ CRPP, Association EURATOM/Confédération Suisse, EPFL, 1015 Lausanne, Switzerland \\ ${ }^{4}$ Department of Physics and Astronomy, EURATOM/VR Fusion Association, PO Box 516, \\ Uppsala University, SE-751 20 Uppsala, Sweden \\ E-mail: ian.chapman@ccfe.ac.uk
}

Received 1 September 2011

Published 28 October 2011

Online at stacks.iop.org/PPCF/53/125002

\begin{abstract}
Sheared toroidal rotation is found to increase the ideal external kink stability limit, thought to be the ultimate performance limit in fusion tokamaks. However, at rotation speeds approaching a significant fraction of the Alfvén speed, the toroidal rotation shear drives a Kelvin-Helmholtz-like global plasma instability. Optimizing the rotation profile to maximize the pressure before encountering external kink modes, but simultaneously avoiding flow-driven instabilities, can lead to a window of stability that might be attractive for operating future high-performance fusion devices such as a spherical tokamak component test facility.
\end{abstract}

\section{Introduction}

A future magnetically confined fusion power plant is predicated upon achieving noninductively sustained plasmas operating at high $\beta_{\mathrm{N}}[1,2]$, where $\beta_{\mathrm{N}}=2 \mu_{0}\langle p\rangle a / B_{0} I_{\mathrm{p}}$ [MA], $\langle p\rangle$ is the volume averaged pressure, $B_{0}$ is the toroidal magnetic field, $a$ is the minor radius and $I_{\mathrm{p}}$ is the plasma current. Previous power plant optimization studies [3] concluded that the cost of electricity scales with $\beta_{\mathrm{N}}^{-0.4}$, making high $\beta_{\mathrm{N}}$ operation attractive. Furthermore, high temperature, and consequently high $\beta_{\mathrm{N}}$, plasmas are also desirable to enhance current drive efficiency and maximize the plasma-generated non-inductive bootstrap current [4].

Modern-day tokamaks heated by neutral beam injection (NBI) can rotate toroidally at a significant fraction of the Alfvén speed [5]. Such strong toroidal rotation can ameliorate or suppress many magnetohydrodynamic (MHD) instabilities, including resistive wall modes $[6,7]$, ballooning modes [8-10] and the internal kink mode [11, 12]. However, the neutral beam driven rotation in ITER and future fusion reactors is likely to be much slower (though the intrinsic plasma rotation is extremely uncertain). Thus, it is important to assess whether 
the toroidal rotation in current devices plays a key role in MHD stability, which may then be absent in ITER. Spherical tokamaks (STs) can have toroidal flows approaching the ion sound speed due to the small plasma volume per unit of NBI power and low moment of inertia of the plasma. Future STs, such as the Component Test Facility (ST-CTF) [13-15], are likely to rotate extremely quickly, with the TRANSP code [16] predicting the core rotation to be approximately equal to the Alfvén speed. At such large sheared rotation velocities, magnetic Kelvin-Helmholtz (KH) instabilities [17] become a concern, as recently observed in a magnetized plasma in the solar corona [18]. In the same way that previous studies have investigated shaping or aspect ratio effects to optimize fusion power plant design [19, 20], here we assess the effects of strong rotation on stability limits in tokamak plasmas. In section 2 we provide details of the numerical tools used and the plasma equilibria studied, before describing the effects of rotation on the pressure limit in section 3. Finally, in section 4 we describe the upper bound to the allowable rotation imposed by $\mathrm{KH}$ instabilities before discussing the implications in section 5 .

\section{Equilibria}

The plasma stability is assessed using the CASTOR-FLOw code [21] using an equilibrium derived from the DIVA code [22], a free-boundary equilibrium code which treats the equilibrium toroidal rotation self-consistently. Whilst treating the rotation only in the stability analysis can be a valid approximation at very sub-Alfvénic rotation velocities, it can also lead to significantly misleading, or even erroneous results $[23,24]$. The stability of over 6000 equilibria with different pressures, toroidal rotation velocities and profiles has been tested. The reference equilibrium is chosen to be an ITER-like case, with pressure and current density profiles as in the ITER baseline scenario [27] with a free-boundary solution constrained by the poloidal field currents and coil positions of ITER. The shape is illustrated in figure 1 together with typical pressure and current profiles. Only a weak pressure pedestal is included and the resultant bootstrap current is not calculated self-consistently for each equilibrium. However, our primary aim is to consider how the rotation affects the pressure limit, and as such this is a somewhat arbitrary equilibrium from which to perform these rotation scans. Instead we varied the $q$-profile, pressure peaking and other global factors and found that the relative increase in beta limit due to the inclusion of rotation in the equilibrium and stability analysis was approximately the same for equilibria $\beta$-limited by $n=1$ external kink instabilities.

The minimum safety factor is scaled by varying the toroidal field and current (whilst $I / a B$ is fixed) to have $q_{\min } \in[1.2,1.7]$ in order to avoid introducing the $q=1$ surface as the rotation is varied. This means that we always consider the same instability as the introduction of $q=1$ would result in unstable internal kink modes too, in addition to which $q_{\min }>1$ is assumed for future power plants [28]. Here $q=\mathrm{d} \psi_{\phi} / \mathrm{d} \psi_{\theta}$ and $\psi_{\phi}$ and $\psi_{\theta}$ are the toroidal and poloidal magnetic fluxes. The reference $q$-profile is taken to have $q_{0}=1.422$ as illustrated in figure 2 . Also overlaid is the eigenfunction of an external kink mode, found to be unstable in these plasmas above a critical plasma beta.

As the rotation is varied, both $I / a B$ and the safety factor on axis are fixed since the $\beta$-limit varies with the former [25] and changes in the latter would result in a variation of the most unstable eigenmode. Since the poloidal field coil currents are also held fixed and the rotation incurs a centrifugal shift of the magnetic axis, this means that $I$ and $B$ are varied slightly. If the safety factor is allowed to vary it is seen that the rotation causes $q_{0}$ to drop, with the decrease dependent upon $\beta$, as illustrated in figure 3 . This can be attributed to the dynamical enhancement of $\beta_{\mathrm{p}}$ by the rotation [26] and a small flow-induced variation in the plasma shape. 

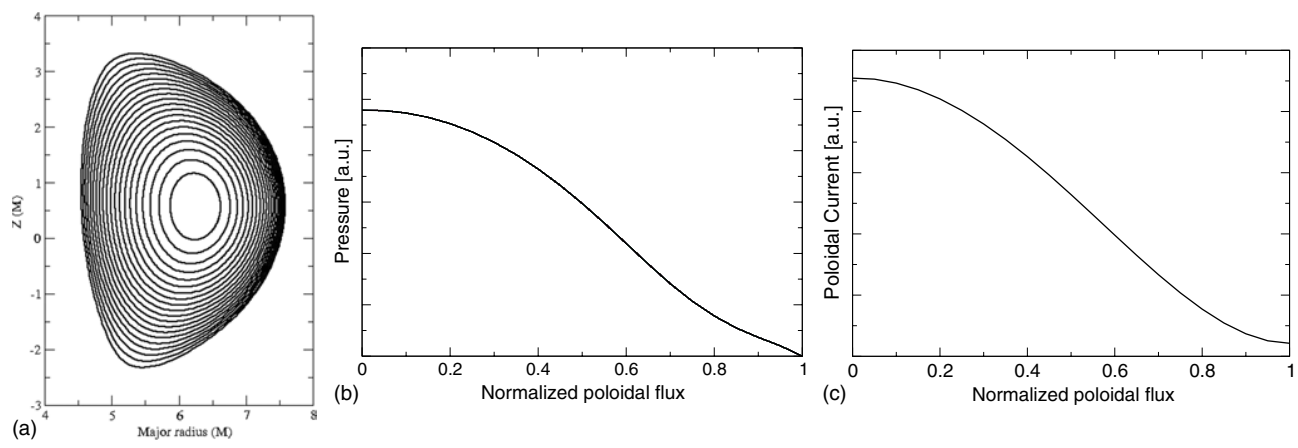

Figure 1. The $(a)$ shape, $(b)$ pressure and $(c)$ poloidal current profile for a typical ITER-like equilibrium with elevated safety factor.

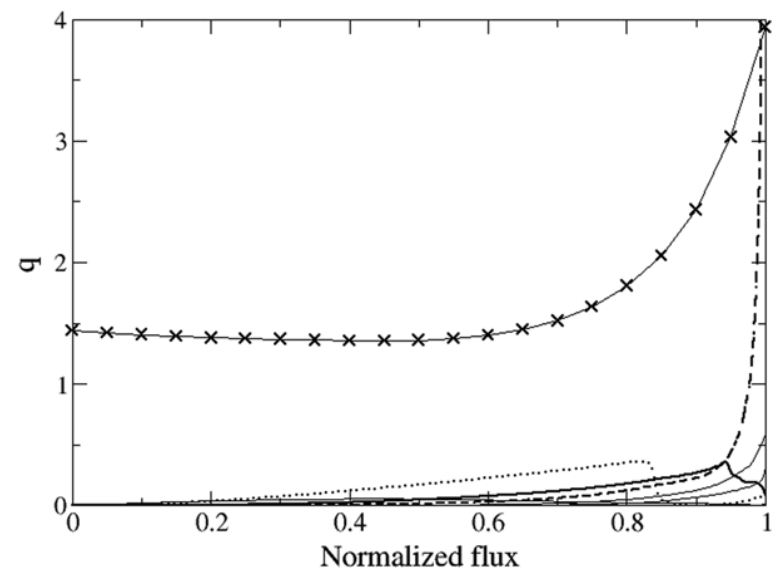

Figure 2. The safety factor profile in the ITER equilibrium with elevated $q$. Overlaid is a typical external kink mode eigenfunction.

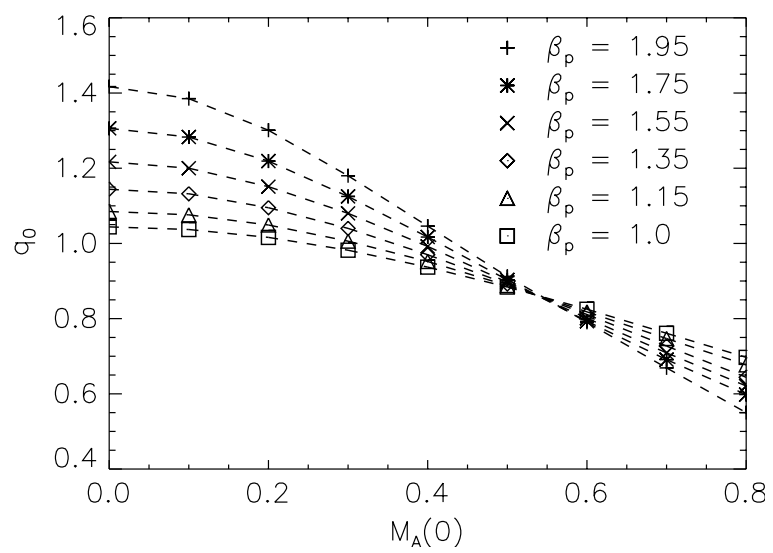

Figure 3. The variation of $q_{0}$ with $\mathcal{M}_{\mathrm{A}}(0)$ for different $\beta_{\mathrm{p}}$ assuming a linearly sheared rotation profile. 


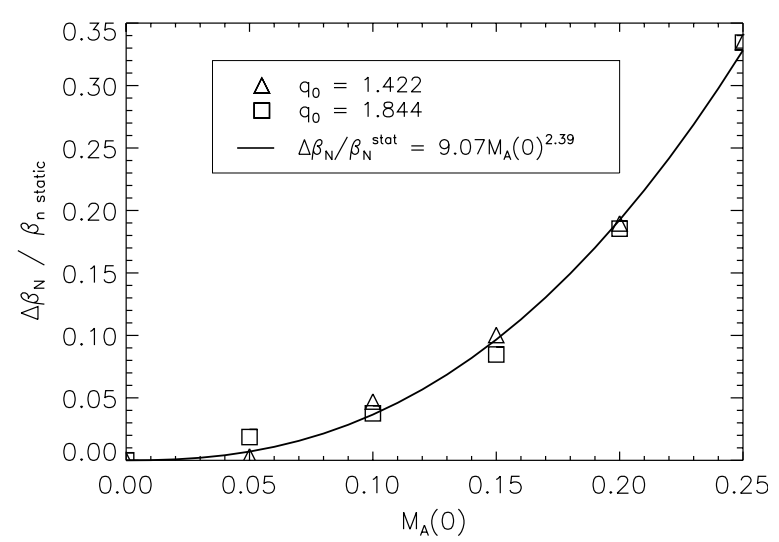

Figure 4. The fractional increase in $\beta$-limit versus $\mathcal{M}_{\mathrm{A}}(0)$ for different $q_{0}$.

\section{The effect of rotation on the pressure limit}

The static $\beta$-limit for the ITER-like case with a fixed $q_{0}=1.4$ is found to be $\beta_{\mathrm{N}}=2.05$, at which point the equilibrium becomes unstable to an external kink (XK) mode. The stability of flowing equilibria is then tested for various toroidal rotation profiles, $\Omega(r)$, with different Alfvénic Mach number at the magnetic axis, $\mathcal{M}_{\mathrm{A}}(0)=\Omega(0) / \omega_{\mathrm{A}}(0)$, and different rotation shears, for increasing plasma pressures. Here the Alfvén frequency is $\omega_{\mathrm{A}}^{2}=B_{0}^{2} / \mu_{0} \rho_{0} R_{0}^{2}, \rho_{0}$ is the mass density and $R_{0}$ is the major radius at the magnetic axis, respectively. The toroidal rotation profile is taken to be of the form $\mathcal{M}_{\mathrm{A}}(\psi)=\mathcal{M}_{\mathrm{A}}(0)\left(a_{0}+a_{1} /\left[1+\exp \left(\left(\psi_{\theta}-a_{2}\right) / a_{3}\right)\right]\right)$ where $a_{0-3}$ are real numbers. This allows a range of rotation profiles, from rigid or linearly sheared to experimental cases or ITER predictions [29]. Figure 4 shows the fractional increase in the $\beta_{\mathrm{N}}$-limit compared with the static case for two different ITER equilibria with $q_{0}=1.42,1.84$ as $\mathcal{M}_{\mathrm{A}}(0)$ is increased for a linearly sheared profile. For rotation speeds of a quarter of the Alfvén speed, which are achievable in present-day STs, the stability limit to the XK mode has increased by a third. The increase in $\beta$-limit is independent of the $q$-profile nuances, provided no ancillary rational surfaces are introduced, or $\Delta q=q_{\min }-1$ does not approach zero, driving infernal modes [30]. It is critical that the equilibrium flow is treated consistently, or the stabilization of the XK mode through dynamic modification of the profiles is not fully captured. Unfortunately it is not possible to produce a $\beta_{\mathrm{N}}$ scaling law for arbitrary rotation profiles and amplitudes because at high rotation speeds KH modes [17] are driven unstable by the flow shear, providing an upper bound on stability. Furthermore, even at modest rotation speeds, the marginally stable $\mathrm{XK}$ mode couples to the $\mathrm{KH}$ mode, making the inference of the pressure limits non-trivial. However, having tested a range of rotation profiles, the linearly sheared profile always has the weakest stabilizing effect on the XK mode, so the 'minimum' increase in the external kink mode stability limit due to sheared rotation (including the modification of the flowing equilibrium) for $R / a=3$ plasmas is given by

$$
\beta_{\mathrm{N}}^{\text {flow }}=\beta_{\mathrm{N}}^{\text {static }}\left(1+9.07 \mathcal{M}_{\mathrm{A}}(0)^{2.39}\right)
$$

provided that the minimum in the safety factor is above the infernal mode threshold (i.e. $q_{\min }$ is well above unity) and that the rotation shear is below the threshold for destabilizing a $\mathrm{KH}$ mode, as discussed in section 4. To use equation (1) for extrapolation to future devices, an expression for $\beta_{\mathrm{N}}^{\text {static }}$ can be taken from scalings with aspect ratio, $I_{\mathrm{p}} / a B$, shaping, pressure peaking, internal inductance and so on, as given in references [19] or [20] for instance. 

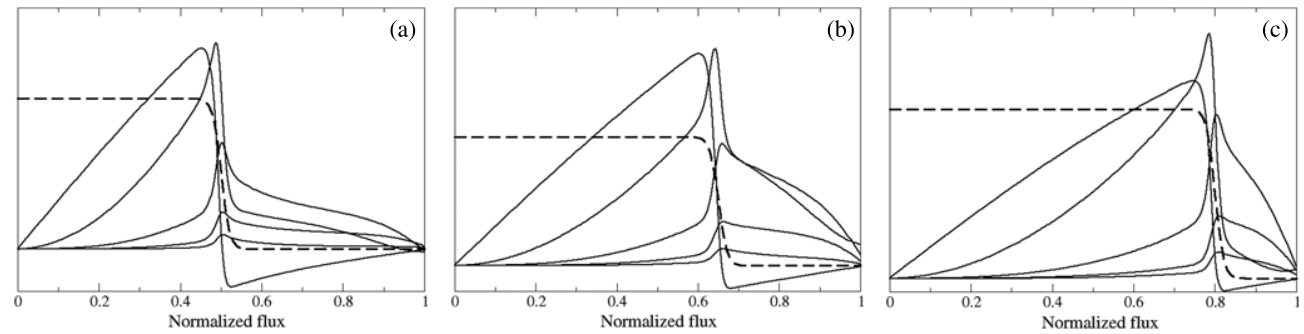

Figure 5. The KH eigenfunctions when the flow profile step is moved radially outwards from $(a)$ $r / a=0.5$ to $(b) r / a=0.65$ to $(c) r / a=0.8$. The flow profile is shown by the dashed lines. The $m=1$ and $m=2$ poloidal harmonics provide the largest components to the perturbation.

\section{KH modes}

KH modes have been an ephemeral concern in tokamak research in the past [31-35] though their empirical absence has allayed this (it should be noted, however, that in NBI-heated plasmas the inherent coupling between rotation and $\beta$ complicates the unambiguous determination of pressure or flow-driven instabilities). However, in future devices, such as the proposed ST-CTF, the rotation is likely to be in excess of present tokamaks. Whilst the toroidal field in designs for an ST-CTF $[13,14]$ increases by a factor of four from present STs resulting in a rise in the Alfvén speed, the plasma density also increases and the injected power goes up an order of magnitude. Numerically, the $\mathrm{KH}$ mode is shown to be driven by the rotation shear. In a large-aspect ratio, circular plasma, $q_{0}=1.3, \beta_{\mathrm{N}}=1.3$ (which is below the pressure limit in the absence of a close-fitting wall) with the rotation profile approximately a Heaviside step function but with finite gradient, the $n=1 \mathrm{KH}$ mode is driven unstable above a critical rotation threshold, in this case $v=0.1 \omega_{\mathrm{A}}$, with a global eigenmode peaked at the position of the step in the rotation. Here $n$ is the toroidal periodicity of the wave. Figure 5 shows the displacement from linear stability analysis for a stepped rotation profile when the position of the step is moved radially outwards. Whilst the position of the step influences the growth rate, it does not change the critical rotation threshold. Notably, even moving the strong flow gradient across a rational surface does not affect the critical rotation to drive the mode. However, as the rotation step crosses a rational surface (in this case at $q=2$ ) the frequency of the mode changes sign, as predicted by cylindrical analysis [31]. The growth rate and frequency dependence of the KH mode are shown in figure 6 . The position of the largest gradient in the rotation thus wholly determines the mode structure, frequency and direction of propagation, but does not strongly affect the growth rate or critical rotation. The KH mode is approximately independent of pressure, and has a finite growth rate as $\beta \rightarrow 0$. Higher toroidal mode number $(n) \mathrm{KH}$ modes usually have larger growth rates, as illustrated in figure 7 , though the eigenstructure for increasing $n$ becomes markedly more radially localized around the step, meaning that such mid- $n$ KH modes are unlikely to represent a significant performance limitation.

The rotation-driven instability can be modelled analytically using the theory for MHD modes in toroidally rotating plasmas developed in [36]. This theory is based on the ordering $\omega \sim \Omega \sim c_{\mathrm{s}} \sim \epsilon \omega_{\mathrm{A}}$ and $\beta \sim \epsilon^{2} \ll 1$ and assumes that there exists an extended region of low magnetic shear ( $s=r / q \mathrm{~d} q / \mathrm{d} r$ ) in the plasma unstable to quasi-interchange modes [37], where $m / q-n \sim \epsilon$. Here $c_{\mathrm{s}}$ is the ion sound speed, $\epsilon=a / R$ is the inverse aspect ratio and $m$ is the poloidal mode number. The equation for a perturbation $\xi_{m, n} \sim \mathrm{e}^{\mathrm{i}(m \theta-n \phi-\omega t)}$ has, in 

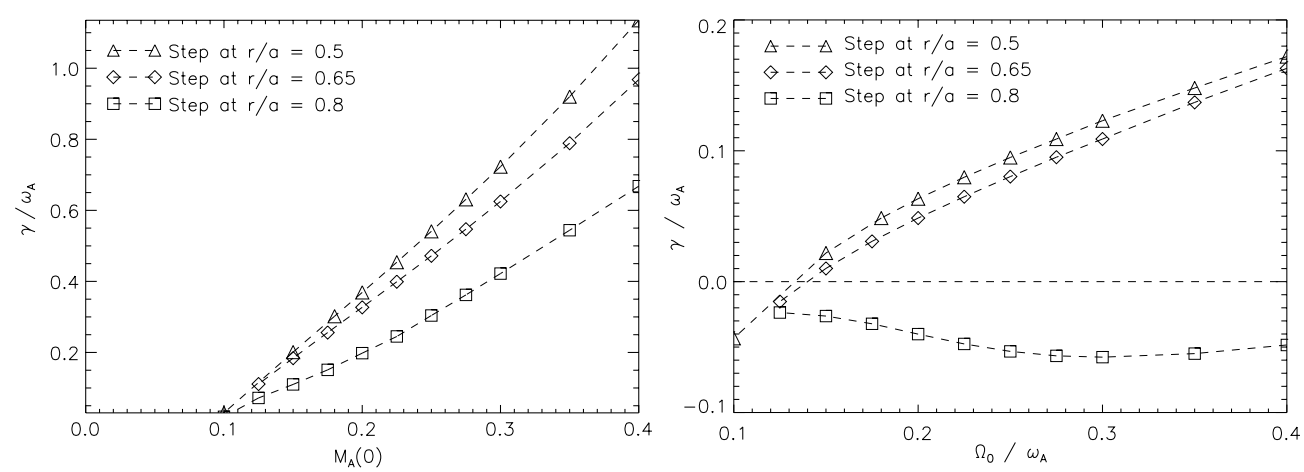

Figure 6. (a) Whilst the critical rotation is unchanged as the step in the flow profile moves radially outside the $q=2$ rational surface, $(b)$ the mode frequency changes sign.

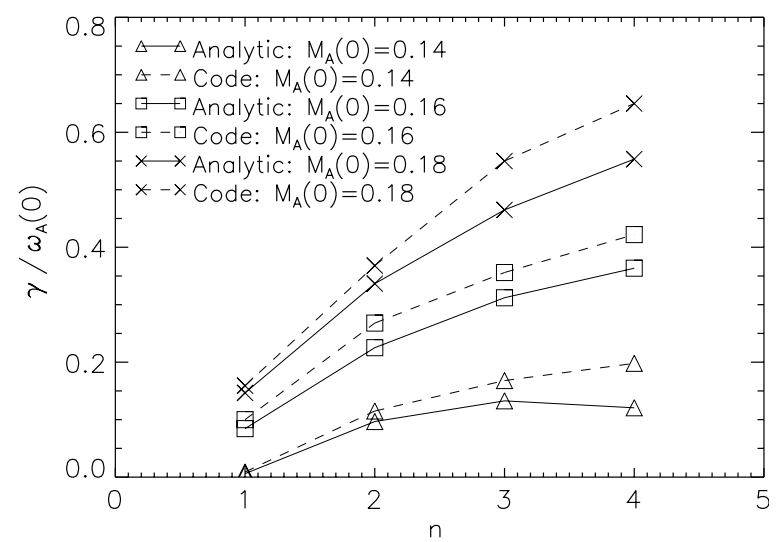

Figure 7. $\mathrm{KH}$ growth rate for different toroidal mode numbers at three $\mathcal{M}_{\mathrm{A}}(0)$ as calculated using the CASTOR-FLOW code and calculated analytically for a circular equilibrium with $\epsilon=0.1$ and $q_{0}=1.1$.

the low-shear region, the form

$$
\begin{aligned}
& \left(\mathcal{L}_{m, n}+\mathcal{T}_{m, n}\right) \xi_{m, n}+r^{2}\left(m^{2}-n^{2}\right) \frac{\mathrm{d} \tilde{\beta}_{0}}{\mathrm{~d} r} \xi_{m, n} \\
& -m^{2} \frac{\mathrm{d} \tilde{\beta_{0}}}{\mathrm{~d} r} C_{+} r^{m+1}=0
\end{aligned}
$$

where $\mathcal{L}_{m, n}$ is the usual cylindrical tokamak operator and $\mathcal{T}_{m, n}$ is an operator including the effects of the plasma inertia as well as the main effects from the plasma rotation [36]. Additional effects of the rotation are, however, also present in $\tilde{\beta}_{0}=2 \mu_{0}\left(p_{0}+\rho_{0} \Omega^{2} R_{0}^{2} / 2\right) / B^{2}$ which represents the 'effective' beta, including both the static pressure and the dynamical pressure from the rotation. The constant $C_{+}$denotes the amplitude of the homogeneous part of the poloidal side-band $\xi_{m+1, n}$ in the low-shear region where equation (2) is valid, and is determined by matching the solution in that region to the solution in the region of large magnetic shear:

$$
C_{+}=-\frac{\Lambda_{m, n} m^{2} R_{0}^{2}}{n^{2} r_{1}^{2 m+2}} \int_{0}^{r_{1}} r^{m+1} \frac{\mathrm{d} \tilde{\beta}_{0}}{\mathrm{~d} r} \xi_{m, n} \mathrm{~d} r
$$

6 

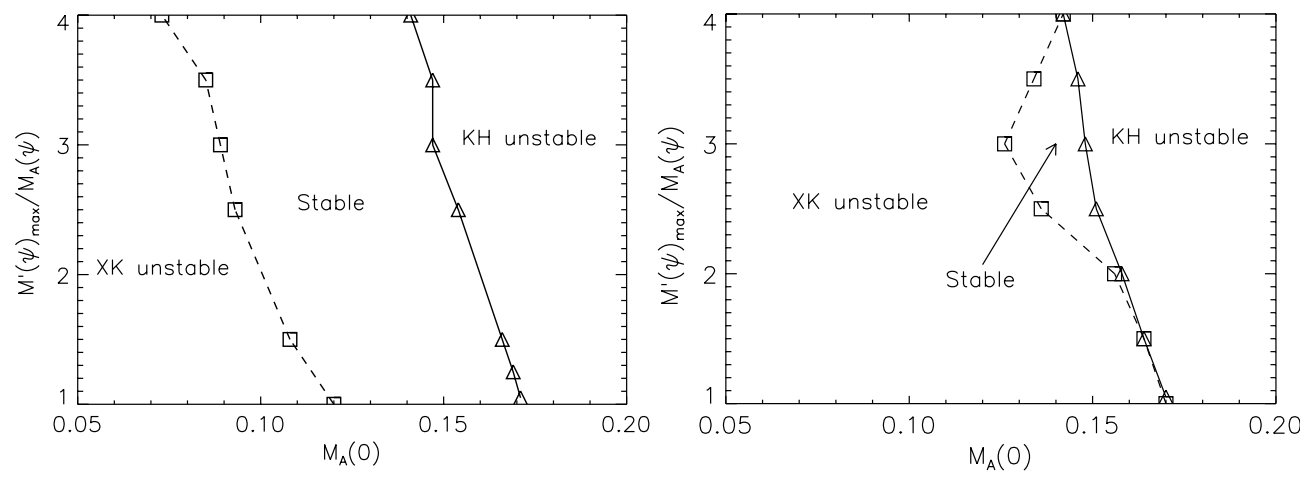

Figure 8. The operational space of rotation shear versus amplitude for the $\mathrm{XK}$ and $\mathrm{KH}$ limits at (left) $\beta_{\mathrm{N}}=2.15$ and (right) $\beta_{\mathrm{N}}=2.25$ for an ITER equilibrium with $q_{0}=1.4$. The $\mathrm{KH}$ limit is almost independent of $\beta_{\mathrm{N}}$.

where $\Lambda_{m, n}=(m+1)(m+2+C) /(2 m-2 C), C=r_{1} \xi_{m+1, n}^{\prime} / \xi_{m+1, n}$ (with $\xi_{m+1, n}^{\prime}$ evaluated at $\left.r=r_{1}+0\right)$ and $r_{1}$ is the radius that separates the region of low shear from the region of high shear [38]. While the middle term in equation (2) represents the stabilizing Mercier effect (for $m>n$ ), the $C_{+}$term usually has the opposite sign (for $\tilde{\beta}_{0}^{\prime}<0$ ) and is the driving term for infernal and quasi-interchange instabilities in static, low-shear plasmas [37,38]. Although a sheared rotation-driven, $\mathrm{KH}$ type of instability is associated already with the homogeneous part of equation (2), it is seen that, since $\tilde{\beta}_{0}$ includes also the dynamical pressure, the rotation shear contributes with an infernal mode drive through the $C_{+}$term, an effect that increases the growth rate of the $\mathrm{KH}$ instability. Good agreement between analytic theory and numerical modelling is illustrated in figure 7 for a large-aspect ratio $\epsilon=0.1$, circular equilibrium with $q_{0}=1.1$ and $\beta=0.005$ and a rotation profile given by $\Omega(r)=\frac{1}{2} \Omega_{0}+\frac{1}{2} \Omega_{0} \tanh \left[\left\{(0.5 a)^{2}-r^{2}\right\} /(0.15 a)^{2}\right]$.

\section{Discussion}

It is possible to examine windows of operating space in terms of rotation amplitude and shear for a given equilibrium $q_{0}$ and $\beta_{\mathrm{N}}$. Using a rotation profile with the maximum shear located at the mid-radius, and varying the parameters $a_{0-3}$ and $\mathcal{M}_{\mathrm{A}}(0)$, a range of flowing free-boundary ITER equilibria with fixed $q_{0}=1.42$ and fixed $I / a B$ have been tested for linear stability to the XK and KH modes. Figure 8 shows the stable operating space for both $\beta_{\mathrm{N}}=2.15,2.25$, which are, respectively, $5 \%$ and $10 \%$ above the ideal no-wall limit. It is evident that increasing the flow above $0.1 \omega_{\mathrm{A}}$ stabilizes the external kink above the no-wall limit. A stronger rotation shear stabilizes the XK mode more effectively than a linearly sheared profile, but at the same time is more likely to drive the $\mathrm{KH}$ mode unstable as the rotation amplitude increases. At $\beta_{\mathrm{N}}=2.25$ the XK mode is found to be more unstable at the highest rotation shear because of a coupling between the XK mode and the marginally stable $\mathrm{KH}$ mode. A window of stability exists with optimized rotation profile and amplitude whereby operation well in excess of the ideal no-wall limit can be achieved. Access above the static pressure limits is replicated in the presence of an ideal wall as well, meaning that a combination of rotation speed and shear allows stable plasmas above the static with-wall stability limits. This may be of importance for future fusion power plants which are predicated upon achieving extremely high plasma pressures [2]. In ITER, however, the rotation speed is predicted to reach only $v_{0} / c_{\mathrm{s}}=0.085$ [29] meaning that the stability limits may be lower than achievable in modern-day tokamaks where strong NBI-induced flows enhance macroscopic stability. 
In contrast, STs can rotate at large fractions of the Alfvén speed. For a typical MAST plasma at low aspect ratio, the critical rotation threshold for $\mathrm{KH}$ onset is calculated to be approximately $0.4 \omega_{\mathrm{A}}$. The upper bound to the plasma rotation or local plasma rotation shear set by the KH mode has significant implications for the operation of an ST-CTF which is likely to rotate at a large fraction of the Alfvén speed if the NBI power required to heat the plasma is unidirectional. Whilst the $\mathrm{KH}$ mode limits the rotation, balancing the beams to avoid $\mathrm{KH}$ modes may then make non-inductive current drive requirements more challenging. The $\mathrm{KH}$ limit is dependent upon the inverse aspect ratio, the wall position and the toroidal mode number as well as the flow amplitude and shear; conversely it is relatively independent of $\beta$ and the adiabatic index. Resistivity, viscosity and kinetic effects are also likely to play a role in $\mathrm{KH}$ mode dynamics.

\section{Conclusions}

Sheared toroidal rotation is found to increase the ideal external kink stability limit, with stronger shear resulting in enhanced stabilization. However, for toroidal rotation approaching a significant fraction of the Alfvén speed, the flow shear drives a Kelvin-Helmholtz global instability, setting an upper limit on the rotation speed for stable plasma operation. Optimizing the rotation profile to maximize the pressure before encountering external kink modes, but simultaneously avoiding flow-driven instabilities, might lead to a window of stability attractive for operating future high-performance high-torque fusion devices, such as next generation spherical tokamaks.

\section{Acknowledgments}

The authors acknowledge useful discussions with C G Gimblett and thank E Strumberger, H P Zehrfeld, C Konz for providing us with the CASTOR-FLOw and DIVA codes. This work was partly funded by the RCUK Energy Programme under grant EP/I501045 and the European Communities under the contract of Association between EURATOM and CCFE. The views and opinions expressed herein do not necessarily reflect those of the European Commission.

Euratom (C) 2011.

\section{References}

[1] Maisonnier D et al 2006 Fusion Eng. Des. 811123

[2] Kessel C E, Mau T K, Jardin S C and Najmabadi F 2006 Fusion Eng. Des. 8063

[3] Ward D J, Cook I and Knight P J 2000 18th IAEA Fusion Energy Conf. (Sorrento, Italy) IAEA-CN-77-FT/P2-20

[4] Bickerton R J, Connor J W and Taylor J B 1971 Nature Phys. Sci. 229110

[5] Helander P, Akers R J and Eriksson L G 2005 Phys. Plasmas 12112503

[6] Gimblett C G 1986 Nucl. Fusion 26617

[7] Garofalo A M et al 2002 Phys. Rev. Lett. 89235001

[8] Cooper W A 1988 Plasma Phys. Control. Fusion 301805

[9] Furukawa M and Tokuda S 2005 Nucl. Fusion 45377

[10] Saarelma S et al 2007 Nucl. Fusion 4931

[11] Waelbroeck F L 1996 Phys. Plasmas 31047

[12] Chapman I T 2011 Plasma Phys. Control. Fusion 53013001

[13] Peng Y K M et al 2005 Plasma Phys Control Fusion 47 B263

[14] Voss G et al 2008 Fusion Eng. Des. 83 1648-53

[15] Stambaugh R D et al 2011 Fusion. Sci. Technol. 59279

[16] Budny R V 1992 Nucl. Fusion 32429 
[17] Kelvin L 1871 Phil. Mag. 42362

[18] Foullon C et al 2011 Astrophys. J. Lett. 729 L8

[19] LinLiu Y R and Stambaugh R D 2004 Nucl. Fusion 44548

[20] Menard J E et al 2003 Unified ideal stability limitd for advanced tokamak and spherical torus plasmas PPPL Report 3779

[21] Strumberger E et al 2005 Nucl. Fusion 451156

[22] Zehrfeld H P 1999 26th European Physical Society Annual Conf. of Plasma Physics (Maastricht, The Netherlands) vol 23 p 1421

[23] Wahlberg C, Chapman I T and Graves J P 2009 Phys. Plasmas 16112512

[24] Chapman I T, Graves J P and Wahlberg C 2010 Nucl. Fusion 50025018

[25] Troyon F et al 1988 Plasma Phys. Control. Fusion 301597

[26] Wahlberg C 2005 Proc. 32nd EPS Conf. on Plasma Physics 29C P4.068

[27] Gribov Y, ITER Organisation 2010 Private Communication

[28] Chapman I T, Kemp R and Ward D J 2011 Fusion Eng. Des. 86141

[29] Budny R V 2009 Nucl Fusion 49085008

[30] Manickam J, Pomphrey N and Todd A M M 1987 Nucl. Fusion 271461

[31] Gimblett C G et al 1996 Phys. Plasmas 33619

[32] Catto P J et al 1973 Phys. Fluids 161719

[33] Mikhailovskii A B 1982 J. Plasma Phys. 281

[34] Chu M S 1995 Phys. Plasmas 5183

[35] Haverkort J W and de Blank H J 2011 Plasma Phys. Control. Fusion 53045008

[36] Wahlberg C 2009 Plasma Phys. Control. Fusion 51085006

[37] Waelbroeck F L and Hazeltine R D 1989 Phys Fluids 1499

[38] Hastie R J and Hender T C 1988 Nucl Fusion 28585 